have roles in the regulation of cell-cell adhe$\operatorname{sion}^{13}-$ is there a part for Shank proteins to play here too?

Despite these open questions, Shank proteins have now become an exciting target for further studies. Furthermore, the combined approach of structural biology and cell biology used by Lilja et al. once again demonstrates the importance of interdisciplinary approaches to tackle fundamental questions underlying human health and disease ${ }^{6}$.

\section{COMPETING INTERESTS}

The authors declare no competing interests.

1. Yan, Z., Kim, E., Datta, D., Lewis, D. A. \& Soderling, S. H. J. Neurosci. 36, 11411-11417 (2016).

2. Monteiro, P. \& Feng, G. Nat. Rev. Neurosci. 18, 147-157 (2017).

3. Phelan, K. \& Rogers, R. C. in GeneReviews (eds Pagon, R. A. et al.) (Univ. Washington, 1993).

4. Peça, J. et al. Nature 472, 437-442 (2011).

5. Duffney, L. J. et al. Cell Rep. 11, 1400-1413 (2015)

6. Lilja, J. et al. Nat. Cell Biol. 19, 292-305 (2017).

7. Case, L. B. \& Waterman, C. M. Nat. Cell Biol. 17, 955-963 (2015).

8. Koser, D. E. et al. Nat. Neurosci. 19, 1592-1598 (2016).
9. Winograd-Katz, S. E., Fässler, R., Geiger, B. \& Legate, K. R. Nat. Rev. Mol. Cell Biol. 15, 273-288 (2014).

10. Horton, E. R. et al. Nat. Cell Biol. 17, 1577-1587 (2015).

11. Przybyla, L., Muncie, J. M. \& Weaver, V. M. Annu. Rev. Cell Dev. Biol. 32, 527-554 (2016).

12. Atherton, P. et al. Nat. Commun. 6, 10038 (2015).

13. Boettner, B. \& Van Aelst, L. Curr. Opin. Cell Biol. 21, 684-693 (2009).

14. Calderwood, D. A., Campbell, I. D. \& Critchley, D. R. Nat. Rev. Mol. Cell Biol. 14, 503-517 (2013).

15. Mameza, M. G. et al. J. Biol. Chem. 288, 26697-26708 (2013).

16. Plak, K., Pots, H., Van Haastert, P. J. M. \& Kortholt, A. BMC Cell Biol. 17, 1 (2016).

\title{
Hippo interferes with antiviral defences
}

\author{
Natalia Muñoz-Wolf and Ed C. Lavelle
}

\begin{abstract}
The Hippo pathway responds to environmental factors including nutrient availability, cell density and substrate stiffness to regulate organ size. This pathway is now shown to also regulate antiviral defence by modulating the TBK1-mediated control of interferon production.
\end{abstract}

Immune responses to pathogens are often investigated in isolation to generate mechanistic insights into innate sensors and protective immune pathways. In the case of viral infections, elegant models have been established, demonstrating the roles of sensors including retinoic acid inducible gene I (RIG-1) for viral RNA and cyclic GMP-AMP synthase (GAS) for viral $\mathrm{DNA}^{1}$. Through engagement with mitochondrial antiviral-signalling protein (MAVS) and stimulator of interferon genes (STING) respectively, this sensing leads to the activation of TANK binding kinase 1 (TBK1) and of inhibitor of nuclear factor kappa-B kinase (IKKe), and subsequently of the transcription factor interferon regulatory factor 3 (IRF3), which stimulates the transcription of type I and type III interferons (IFNs) and IFNstimulated genes (ISGs) (Fig. 1a). In addition to this core pathway, other factors including nutritional status, the microbiome and cellular metabolism significantly impact on immune

Natalia Muñoz-Wolf and Ed C. Lavelle are in the Adjuvant Research Group, School of Biochemistry and Immunology, Trinity Biomedical Sciences Institute, Trinity College Dublin, Dublin 2, D02 R590, Ireland. Ed C. Lavelle is also at the Centre for Research on Adaptive Nanostructures and Nanodevices (CRANN) and the Advanced Materials Bio-Engineering Research Centre (AMBER), Trinity College Dublin, Dublin 2, D02 PN40, Ireland. e-mail: lavellee@tcd.ie regulation ${ }^{2}$. In this issue, Zhang et al. ${ }^{3}$ unveil a new layer of complexity, showing that the Hippo pathway, which is regulated by the nutrient and physical environment of cells, can serve as a potent regulator of IFN production and antiviral defence.

The Hippo pathway, which is highly conserved in organisms ranging from Drosophila to mammals, controls tissue homeostasis by regulating cell differentiation, proliferation and apoptosis. Core components of the mammalian Hippo pathway include the mammalian sterile 20-like 1 kinases MST1 and MST2, large tumour suppressor kinase 1 and 2 (LATS1 and LATS2), and the adaptors salvador family WW domain containing protein 1 (SAV1) and MOB kinase activator $1 \mathrm{~A}$ and $\mathrm{B}$ (MOB1A and MOB1B). Canonical activation of the Hippo pathway leads to phosphorylation and inhibition of yes-associated protein, YAP, and transcriptional coactivator with PDZ-binding motif, TAZ, preventing their nuclear translocation and interaction with the transcription factors TEA domain family members 1-4 (TEAD1-4; ref. 4; Fig. 1b). In addition to multiple cues for activation, an extensive Hippo pathway interactome has been characterized ${ }^{5}$, indicating significant cross-talk with other pathways. Besides its role in development, Hippo has been implicated in modulation of immune responses. In
Drosophila, the Hippo pathway modulates the transcription of the inhibitor Cactus, the I $\mathrm{B}$ homolog, controlling Toll-mediated innate responses against Gram-positive bacteria ${ }^{6}$. In addition, activation of the Hippo pathway through cell-cell contact during proliferation of antigen-specific effector $\mathrm{CD} 8^{+} \mathrm{T}$ cells triggers expression of the transcriptional repressor, PRDM1 (also known as BLIMP1), inducing terminal differentiation of effector cells ${ }^{7}$, a crucial event for balancing progression from clonal expansion to effector differentiation during antiviral responses.

The study by Zhang et al. ${ }^{3}$ outlines a previously unappreciated role for Hippo in the regulation of antiviral responses, and more specifically in the regulation of type I IFN production. Using starvation and modulation of cell density as canonical activators of the Hippo pathway, they demonstrated that Hippo amplifies Sendai virus (SeV)-induced IRF3 transactivation. Importantly, this starvation-induced effect on IRF3 also applied in the case of ectopic expression of activated STING or RIG-I, suggestive of a broad regulation of RNA- and DNA-sensing pathways. Deletion of LATS1/2, the kinases upstream of YAP/TAZ, abolished the enhancing effects of serum starvation on IRF3 activation. The authors further demonstrated that YAP/TAZ interacted with TBK1, thereby preventing its 


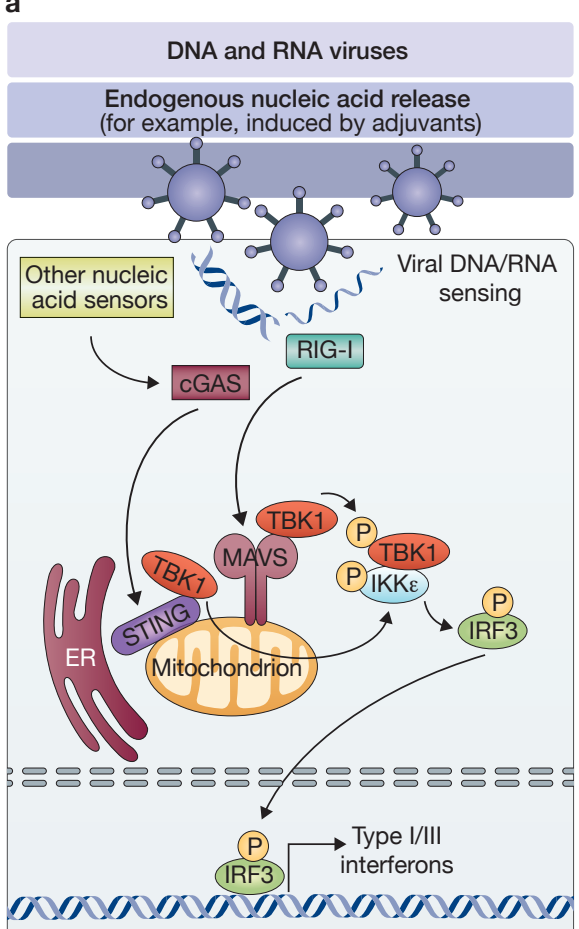

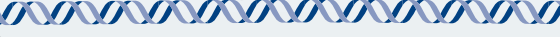

C Starvation,

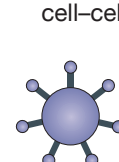

20ell-cell
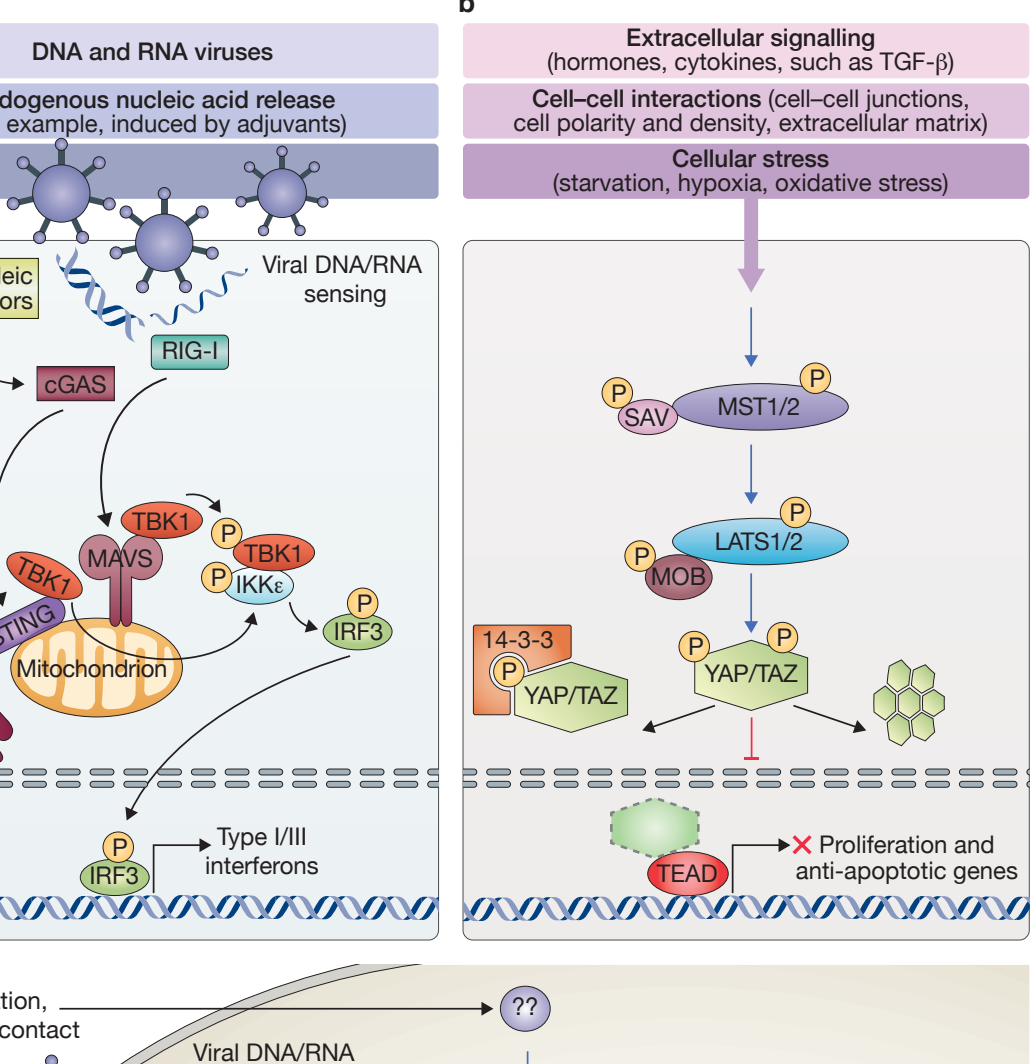

\section{(O)}
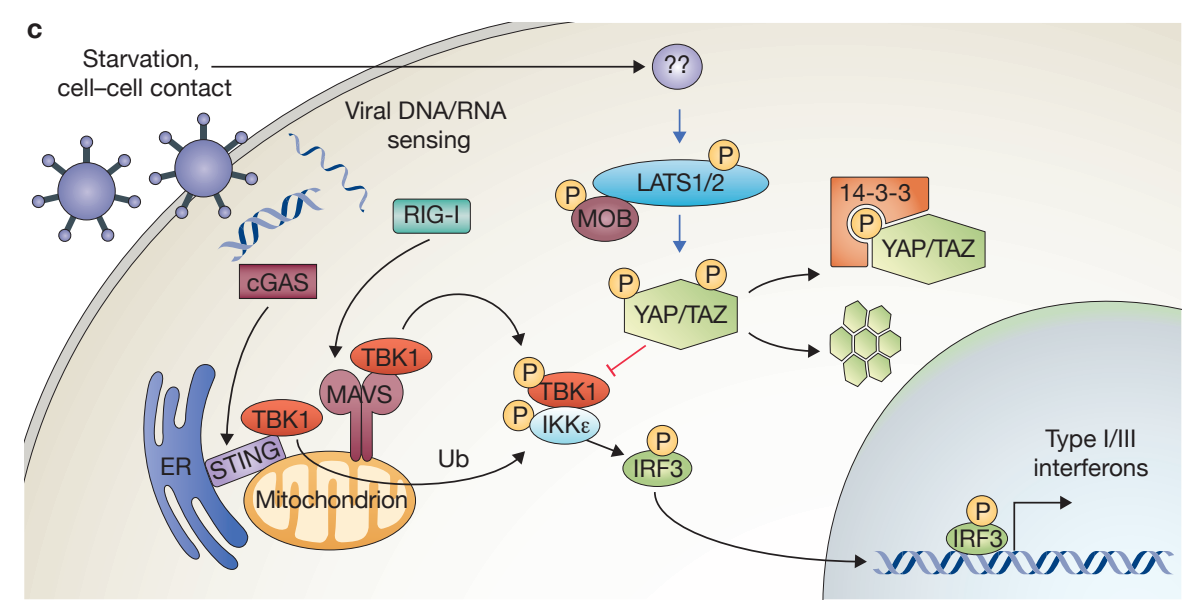

Figure 1 Hippo-YAP regulation of TBK1-mediated antiviral defences. (a) Nucleic acids of viral or other origins that gain access to the cytoplasm are sensed by various cytosolic innate receptors. Viral RNA is sensed by receptors such as RIG-I, which activate MAVS. DNA sensors, such as CGAS, detect cytosolic DNA and engage the STING adaptor protein. These pathways activate the TBKI and IKK $\varepsilon$ kinases, resulting in phosphorylation of IRF3, a member of the IFN regulatory transcription factor family, to promote expression of type I IFNs and other pro-inflammatory cytokines. ER, endoplasmic reticulum. (b) Activation of the Hippo pathway triggers sequential phosphorylation events (represented as blue arrows) by the MST1/2 and LATS1/2 kinases in conjunction with their respective adaptor proteins SAV1 and MOB1A/B leading to the phosphorylation and inactivation of the YAP/TAZ transcriptional coactivators through degradation or cytoplasmic sequestration by 14-3-3 proteins (black arrows). This prevents YAP/TAZ nuclear accumulation and association with the TEAD1-4 transcription factors (depicted by red bar-headed line), and inhibits the transcription of proliferative and anti-apoptotic genes. (c) In the model proposed, by Zhang et al. ${ }^{3}$, YAP/TAZ associate with the TBK1 and IKK $\varepsilon$ kinases in the cytoplasm, blocking their ubiquitination ( $\mathrm{Ub}$ ) and activation and preventing type I IFN production in response to viral nucleic acid sensing. Activation of the Hippo pathway by starvation or cell-cell contact induces YAP/TAZ phosphorylation and its subsequent degradation allows TBK1 association with IKKE, to activate IRF3mediated expression of type I IFN genes.

K63 ubiquitination and subsequent activation (Fig. 1c). Under resting conditions, YAP/TAZ were present in the nucleus, but following vesicular stomatitis virus (VSV) infection, the proteins relocalized in the cytoplasm. Using knockdown experiments and altering cellular conditions to activate the Hippo pathway, the authors demonstrated that Hippo activation abrogated the inhibitory effect of YAP/TAZ on TBK1 and enhanced antiviral responses in a zebrafish model. Expression of transcriptionally inactive human YAP sensitized cultured cells to VSV infection, and ectopic expression in zebrafish resulted in higher mortality and reduced expression of IFNs and ISGs, following VSV challenge. Using an inducible YAP model, the authors showed that induction of YAP led to enhanced replication of GFPtagged VSV or the DNA virus herpes simplex virus 1 (HSV-1). VSV-induced TBK1 activation in colon carcinoma cells was negatively regulated by YAP/TAZ. Although these data are suggestive of a significant role for Hippo signalling in responses to viral infection, it will be important to expand these studies to other animal models of infection and to human settings to assess their broader significance in regulating antiviral immunity.

The inhibition of IRF3 transactivation by YAP was independent of its effects on transcription as shown by using a transcriptionally inactive variant (YAP 6SA). Rather, this was due to the direct interaction of its C-terminal domain with TBK1, which prevented TBK1 K63 ubiquitination, activation, and IRF3 binding. This inhibitory effect was not selective for the IRF3 interaction, as TAZ and the transcriptionally inactive YAP variant also disrupted the interaction of TBK1/IKK $\varepsilon$ with STING and MAVS, indicating that YAP/TAZ block the interaction of these kinases with their substrates and adaptors. Since YAP/TAZ can promote cell proliferation and inhibit apoptosis ${ }^{8,9}$, it will be interesting to assess whether Hippo activation may also regulate virally induced cell death triggered by the presence of cytoplasmic nucleic acids ${ }^{10}$ as a means to control excessive inflammation and pathology.

Although the authors suggest that YAP/TAZ proteins are expressed at a low level in immune cells (THP1 monocytes and peritoneal macrophages), they showed that TRIF-induced IRF3 activation and MYD88-induced NFKB activation were negatively regulated by YAP/TAZ in HEK293 cells. A recent report demonstrated that Mycobacterium tuberculosis activated the Hippo pathway in macrophages, enhancing expression of the chemokines CXCL1/2 (ref. 11). This offers the tantalizing possibility that the Hippo pathway also regulates nucleic-acid-sensing pathways by 
immune cells. Given that TBK1-mediated IRF3 phosphorylation requires adaptor proteins such as MAVS, STING, or TRIF, which must also undergo TBK1 phosphorylation ${ }^{12}$, and in light of the cross-regulation between type I IFN and inflammasome signalling ${ }^{13}$, it will also be of interest to assess whether Hippo signalling impacts on inflammasome activation.

A recent report implicated LATS1/2 in suppressing anti-tumour immunity, by demonstrating that LATS1/2 deletion in tumour cells enhanced the release of nucleic-acidcontaining extracellular vesicles, which promoted a type I IFN response via MYD88/TRIF signalling, enhancing anti-tumour immune responses ${ }^{14}$. Together with the study by Zhang et al., this indicates that Hippo signalling can regulate type I IFN responses through different mechanisms. Given that STING is an attractive target for vaccine adjuvants ${ }^{15}$ and has a role in anti-tumour immunity ${ }^{16}$, it will be interesting to test whether the Hippo activation status has beneficial or detrimental consequences for adjuvants targeting STING and other nucleic acid sensors for both infectious disease and tumour vaccine efficacy. In this context, the unanticipated demonstration of cross-regulation between two evolutionarily conserved and fundamental cellular signalling pathways may have broad implications, as targeted manipulation of the balance between Hippo signalling and IFN responses could have therapeutic potential in fields ranging from immunotherapy to bioengineering and vaccine development.

\section{COMPETING FINANCIAL INTERESTS}

The authors declare no competing financial interests.
1. Zevini, A., Olagnier, D. \& Hiscott, J. Trends Immunol. 38, 195-205 (2017).

2. Pfeiffer, J. K. \& Virgin, H. W. Science $\mathbf{3 5 1}$, aad5872 (2016).

3. Zhang, Q. et al. Nat. Cell Biol. 19, 362-374 (2017).

4. Hansen, C. G., Moroishi, T. \& Guan, K.-L. Trends Cell Biol. 25, 499-513 (2015).

5. Plouffe, S. W. et al. Mol. Cell 64, 993-1008 (2016).

6. Liu, B. et al. Cell 164, 406-419 (2016).

7. Thaventhiran, J. E. D. et al. Proc. Natl Acad. Sci. USA 109, E2223-E2229 (2012).

8. Yu, F.-X., Zhao, B. \& Guan, K.-L. Cell 163, 811-828 (2015).

9. Huang, J., Wu, S., Barrera, J., Matthews, K. \& Pan, D. Cell 122, 421-434 (2005).

10. Fernandes-Alnemri, T., Yu, J.-W., Datta, P., Wu, J. \& Alnemri, E. S. Nature 458, 509-513 (2009).

11. Boro, M., Singh, V. \& Balaji, K. N. Sci. Rep. 6, 37695 (2016).

12. Liu, S. et al. Science 347, aaa2630 (2015).

13. Guarda, G. et al. Immunity 34, 213-223 (2011).

14. Moroishi, T. et al. Cell 167, 1525-1539.e17 (2016).

15. Carroll, E. C. et al. Immunity 44, 597-608 (2016).

16. Deng, L. et al. Immunity 41, 843-852 (2014). 